\title{
A INFLUÊNCIA DA TIPOGRAFIA NA USABIILIDADE: UMA REVISÃO SISTEMÁTICA PRELIMINAR DA LITERATURA
}

\section{THE INFLUENCE OF TYPOGRAPHY IN USABILITY: A PRELIMINARY SYSTEMATIC REVIEW OF LITERATURE}

\author{
Ricardo Jessé Santana da Costa ${ }^{1}$, Me. \\ Lívia Flávia de Albuquerque Campos ${ }^{2}$, Dra. \\ Ana Beatriz Avelar Nascimento ${ }^{3}$, estudante. \\ (1) Universidade Federal do Maranhão \\ e-mail: ricardojesse@gmail.com \\ (2) Universidade Federal do Maranhão \\ e-mail:liviaflavia@gmail.com \\ (3) Universidade Federal do Maranhão \\ abeatrizavelar@gmail.com
}

Tipografia, usabilidade, revisão sistemática.

Este artigo tem como objetivo apresentar o resultado de uma revisão sistemática da literatura nas bases e Science Direct que buscou estudos que correlacionaram tipografia com a usablidade em dispositivos de interação móvel, no período de 2007 até os dias atuais. Como resultado obteve-se que nenhum dos estudos efetivamente utiliza dispositivos móveis em suas pesquisas.

Typography, usability, literature review

This article aims to present the result of a systematic review of literature in the and Science Direct database that sought studies that correlated typography with usability in mobile interaction devices, since 2007 until nowadays. As results it was found that none of the studies actually used mobile devices in their research.

\section{Introdução}

Uma característica marcante no estudo da tipografia reside no forte apelo que um tipo de letra pode estabelecer entre o conteúdo e o leitor, assim como cita Bringhurst (2011, p. 23): o objetivo da tipografia consiste em representar e fortalecer visualmente o conteúdo de uma determinada mensagem.

Sendo assim, designers devem desenvolver um alto nível de conhecimento sobre tipografia, a fim de construir mensagens que não só atraiam leitores, mas permita-lhes ler com facilidade e entender a mensagem criada (BIX, 2002, não paginado).

Com a evolução dos processos de renderização e da produção tipográfica digital, tornou-se ainda mais complexo organizar e estruturar massas textuais visando a transmissão de conteúdo através das mídias digitais. Para Farias (2013, p. 15) as novas tecnologias enfatizaram a relatividade dos limites entre os momentos de criação de uma fonte nova e seu uso. A autora aborda o tema como difícil para o designer gerenciar essa estrutura tipográfica, visto que as novas mídias possibilitam 


\section{$16^{\circ}$ \\ ERGODESIGN USIHC CINAHPA}

que qualquer usuário manipule, distorça uma fonte disponível.

Segundo Hoober \& Berkman (2011, p. 491) é cada vez mais desafiador para os designers adequar a variedade e disponibilidade de tipos, variações de cor, contraste e tamanho ao avanço dos suportes existentes.

Tal cenário pode se tornar de extrema dificuldade para o designer visto que a evolução da produção de conteúdos para visualização em telas de dispositivos móveis se tornaram de extrema relevância no dias de hoje, conforme pontuaram Hammerschmidt \& Spinillo (2014, p. 2).

Em paralelo, embora as ferramentas digitais tenham ampliado os limites da tipografia, ainda há poucos pesquisadores dedicados a investigar essa temática no Brasil, conforme aponta Gomes (2010, p. 15).

Sendo assim, o objetivo deste artigo é apresentar as evidências que correlacionam os estudos, realizados nos últimos 10 anos, sobre tipografia e usabilidade em dispositivos de interação móvel já publicadas em revistas científicas, visando compreender como as pesquisas abordam estas duas áreas do conhecimento.

\section{Fundamentação teórica}

Neste capítulo serão apresentados os principais conceitos sobre esta pesquisa.

\subsection{Tipografia}

É muito comum relacionar tipografia apenas à prática do desenho de tipos. De acordo com Farias (2013, p. 18), tipografia é "o conjunto de práticas subjacentes à criação e a utilização de símbolos visíveis relacionados aos caracteres ortográficos (letras) e para-ortográficos (tais como números e sinais de pontuação) para fins de reprodução".

A tipografia, assim como diversas áreas do conhecimento aplicado ao Design, vem ganhando novos valores conforme as tecnologias de suporte oferecem aos indivíduos a capacidade de interferir na apresentação e prática tipográfica. A fim de estabelecer o quão importante está sendo essa evolução tecnológica para o uso da tipografia $16^{\circ}$ Ergodesign - Congresso Internacional de Ergonomia e Usabilidade de Interfaces Humano Tecnológica: Produto, Informações Ambientes Construídos e Transporte

$16^{\circ}$ USIHC - Congresso Internacional de Ergonomia e Usabilidade de Interfaces Humano Computador

CINAHPA | 2017 - Congresso Internacional de Ambientes Hipermídia para Aprendizagem. adequadamente a estes novos suportes, é necessário definir alguns conceitos inerentes a esta área do conhecimento visando obter o panorama de como os tipos em tela podem melhorar a experiência de uso de dispositivos de interação móvel pelos indivíduos.

Segundo Farias (2013, p.15) "se tipografia contemporânea está ligada à emergência da figura do tipógrafo na condição de indivíduo capaz de redefinir a prática tipográfica com base na inovação é possível dizer que novas tecnologias". Para este fim, o termo tipografia descreveria apenas manifestações da linguagem visível, assim seriam mais propriamente definidas como caligrafia, ou escrita manual, letreiramento (do inglês lettering). Nesse ponto vale ressaltar que informações textuais longas devem ter a atenção dobrada no que diz respeito a articulação tipográfica, ou seja, como textos mais extensos serão apresentados sem que haja perda de informação ou não entendimento por parte do leitor/usuário, segundo afirma Hammerschmidt (2014, p. 26).

Para Hammerschmidt (2014, p. 36) muitas das questões que envolvem os aspectos funcionais da tipografia se encontram relacionadas aos conceitos de legibilidade e leiturabilidade, que constitui um debate antigo na literatura sobre tipografia.

Quando as formas de diferentes letras de um mesmo desenho de tipo podem ser discriminadas com rapidez, diz-se que este tipo é altamente legível, afirma Niemeyer (2010, p. 82). Sendo assim legibilidade está na habilidade visual que um conjunto de caracteres possui de se distinguir internamente, ou seja, serem legíveis.

Com base nessa informação entende-se que a legibilidade de um texto é o que caracteriza o parâmetro para ser avaliado em aplicações quanto a usabilidade que ela proporciona a determinada interface.

Segundo Bastien \& Scapin (1993, p. 16) a legibilidade relaciona-se com o usuário pois possui características que podem dificultar ou facilitar a leitura das informações textuais. Sobre estes aspectos, os pesquisadores afirmam que a 


\section{$16^{\circ}$ \\ ERGODESIGN USIHC CINAHPA}

$16^{\circ}$ Ergodesign - Congresso Internacional de Ergonomia e Usabilidade de Interfaces Humano Tecnológica: Produto, Informações Ambientes Construídos e Transporte

$16^{\circ}$ USIHC - Congresso Internacional de Ergonomia e Usabilidade de Interfaces Humano Computador

CINAHPA | 2017 - Congresso Internacional de Ambientes Hipermídia para Aprendizagem.

legibilidade não está relacionada com feedback ou mensagens de erro dos sistemas e sim a informação textual longa e contínua.

Alguns parâmetros são determinantes para se estabelecer uma boa legibilidade. Tais parâmetros são estabelecidos por diversos autores que atribuem a cada um deles seu valor de importância para garantir a um texto que este seja legível.

Apresenta-se a seguir um quadro-resumo sobre os parâmetros tipográficos:

\begin{tabular}{|c|c|c|}
\hline Item & Definição & Aplicação \\
\hline 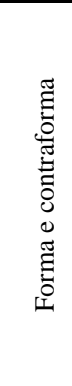 & $\begin{array}{l}\text { A forma, a parte } \\
\text { impressa dos caracteres } \\
\text { é o que não vemos, pois } \\
\text { é preta e implica numa } \\
\text { interrupção de nossa } \\
\text { visão. A contraforma, } \\
\text { por sua vez, é o que } \\
\text { vemos, o branco não } \\
\text { impresso do papel que } \\
\text { reflete luz. (BUGGY, } \\
\text { 2007, p. 93) }\end{array}$ & $\begin{array}{l}\text { A oposição fundamental entre } \\
\text { forma e contraforma } \\
\text { demonstra relevância para a } \\
\text { compreensão de diversos } \\
\text { fatores que influenciam a } \\
\text { legibilidade do texto. Entre } \\
\text { tais fatores, observa-se essa } \\
\text { relação de maneira mais clara } \\
\text { ao se abordar espaçamento, } \\
\text { kerning e tracking. } \\
\text { (HAMMERSCHMIDT, 2014, } \\
\text { p. 38) }\end{array}$ \\
\hline 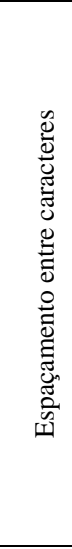 & $\begin{array}{l}\text { a. O kerning é o } \\
\text { ajustamento de espaços } \\
\text { horizontais entre pares } \\
\text { de caracteres } \\
\text { específicos em um } \\
\text { texto, para criar } \\
\text { visualmente um espaço } \\
\text { aparentemente } \\
\text { homogêneo entre todos } \\
\text { os tipos. (NIEMYER, } \\
\text { 2010, p. 73) } \\
\text { b. define como o } \\
\text { controle do espaço } \\
\text { médio entre caracteres } \\
\text { num mesmo bloco de } \\
\text { texto (NIEMYER, } \\
\text { 2010, p. 74) }\end{array}$ & $\begin{array}{l}\text { a. Caracteres muito próximos } \\
\text { tendem a ser lidos como um } \\
\text { só, caracterizando uma baixa } \\
\text { legibilidade mesmo em um } \\
\text { corpo tipográfico maior. } \\
\text { (BRINGHURST, 2011, p. 40) } \\
\text { b. Com o corpo de oito pontos } \\
\text { ou menos, por exemplo, os } \\
\text { tipos não suportam o tracking } \\
\text { em níveis "apertado e muito } \\
\text { apertado", pois assim o texto } \\
\text { tende a ilegibilidade } \\
\text { (NIEMYER, 2010, p. } 74 \text { ) }\end{array}$ \\
\hline 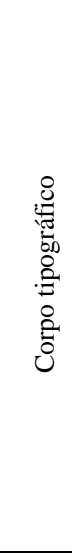 & $\begin{array}{l}\text { É altura máxima do } \\
\text { conjunto de caracteres } \\
\text { em uma fonte, incluindo } \\
\text { as áreas reservadas para } \\
\text { os caracteres mais altos } \\
\text { e mais baixos (FARIAS, } \\
2004 \text {, não paginado) }\end{array}$ & $\begin{array}{l}\text { - As diretrizes encontradas na } \\
\text { literatura especificam faixas } \\
\text { de tamanhos variando entre } \\
8 \text { e } 14 \text { pt como valores } \\
\text { ideais para textos de leitura } \\
\text { contínua (WAARDE (1999, } \\
\text { p. 70); } \\
\text { - Em dispositivos móveis, os } \\
\text { tipos podem ser menores do } \\
\text { que em um computador de } \\
\text { mesa, porque o usuário } \\
\text { pode ajustar a distância } \\
\text { entre a tela e seu rosto para } \\
\text { obter o tamanho ideal para a } \\
\text { leitura (REICHENSTEIN, } \\
\text { 2012, não paginado, } \\
\text { tradução nossa). }\end{array}$ \\
\hline
\end{tabular}

\begin{tabular}{|c|c|c|}
\hline 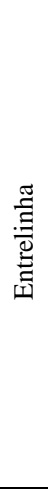 & $\begin{array}{l}\text { Entrelinha ou } \\
\text { entrelinhamento é a } \\
\text { distância de um linha } \\
\text { tipográfica para a outra } \\
\text { (LUPTON, 2013, p. } \\
\text { 104). }\end{array}$ & $\begin{array}{l}\text { - Entrelinhamentos menores } \\
\text { que o corpo causam colisão } \\
\text { entre as ascendentes e } \\
\text { descendentes dos tipos ao } \\
\text { ponto que linhas muito } \\
\text { distantes podem ser } \\
\text { consideradas elementos } \\
\text { gráficos independentes } \\
\text { (LUPTON, 2013, p. 104) } \\
\text { - A adição de } 2 \text { pts à altura } \\
\text { do corpo garante um } \\
\text { entrelinha satisfatório para a } \\
\text { legibilidade } \\
\text { (BRINGHURST, 2011, p. } \\
\text { 45) }\end{array}$ \\
\hline 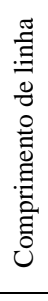 & $\begin{array}{l}\text { Trata-se da quantidade } \\
\text { letras ou caracteres por } \\
\text { linha de texto. }\end{array}$ & $\begin{array}{l}\text { - Linhas de texto com } 40 \text { a } 50 \\
\text { caracteres, destinado a } \\
\text { dispositivos móveis } \\
\text { (HOOBER \& BERKMAN, } \\
\text { 2011, p. 501) } \\
\text { - Na língua portuguesa o } \\
\text { ideal é entre } 10 \text { e } 11 \\
\text { palavras por linha } \\
\text { (HASLAM, 2010, p. 79) }\end{array}$ \\
\hline 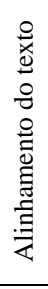 & $\begin{array}{l}\text { O posicionamento do } \\
\text { texto dentro das } \\
\text { margens da página pode } \\
\text { ser com alinhamento à } \\
\text { esquerda ou à direita, } \\
\text { centralizado ou } \\
\text { justificado. } \\
\text { (NIEMEYER, 2010, p. } \\
\text { 77) }\end{array}$ & $\begin{array}{l}\text { Com o alinhamento à } \\
\text { esquerda, textos tendem a } \\
\text { atribuir maior legibilidade, } \\
\text { pois facilita o processo de } \\
\text { mudança de linha em } \\
\text { composições } \\
\text { extensas.(HOOBER \& } \\
\text { BERKMAN, 2011, p. 501) }\end{array}$ \\
\hline
\end{tabular}

Quadro 1 - Resumo sobre parâmetros da articulação tipográfica

\subsection{Usabilidade}

Para tornar a compreensão e correlação da tipografia e sua articulação com a usabilidade é necessário apresentar, à luz das referências prévias sobre o tema, tópicos e conceituações a fim de criar uma base teórica pertinente ao desenvolvimento desta pesquisa.

Segundo Cybis, Betiol \& Faust (2010, p. 16) a usabilidade é qualidade que caracteriza o uso dos programas/sistemas e suas aplicações. Trata-se de uma característica intrínseca de um sistema, porém dependente de um acordo entre as características dos usuários e os aspectos formais das interfaces. Os autores seguem apontando que esta qualidade (usabilidade) pode ser satisfatória para usuários mais habituados com tal sistema e deixar muito a desejar quando, este mesmo sistema, for usado por usuários menos experientes ou novatos.

Já a norma ISO 9241 utiliza a definição de usabilidade como sendo a capacidade que um 


\section{$16^{\circ}$ \\ ERGODESIGN USIHC CINAHPA}

$16^{\circ}$ Ergodesign - Congresso Internacional de Ergonomia e Usabilidade de Interfaces Humano Tecnológica: Produto, Informações Ambientes Construídos e Transporte

$16^{\circ}$ USIHC - Congresso Internacional de Ergonomia e Usabilidade de Interfaces Humano Computador

CINAHPA | 2017 - Congresso Internacional de Ambientes Hipermídia para Aprendizagem. sistema interativo oferece a seu usuário, em determinado contexto de operação, para a realização de tarefas de maneira eficaz, eficiente e agradável.

De acordo com Nielsen (2012, não paginado) a usabilidade é definida por 5 componentes de qualidade em visão ampliada do que a norma ISO 9241 que define como características de um sistema com boa usabilidade. São elas:

1. Apreensibilidade: relaciona-se com o quão fácil é para os usuários a realizar tarefas básicas na primeira vez que se deparam com a interface;

2. Eficiência: trata-se da rapidez com que os usuários podem executar tarefas, uma vez que eles aprenderam sobre a interface;

3. Memorabilidade: ao passar algum tempo sem utilizar o sistema, o usuário é capaz de facilmente reestabelecer proficiência;

4. Erros: relaciona-se com os erros que os usuários executam durante a tarefa, quão grave são esses erros, e como facilmente se pode recuperar;

5. Satisfação: o quão agradável é utilizar o sistema/interface.

\subsection{Dispositivos de interação móvel}

A fim de se estabelecer quais estudos foram adotados como resultado da revisão aqui proposta faz-se necessário a definição de dispositivos de interação móvel - DIM.

Zhang \& Adipat (2005, p. 295) apresentam características únicas dos dispositivos móveis que devem consideradas ao se examinar a usabilidade em tais equipamentos:

1. Contexto móvel: Ao usar aplicações móveis o usuário não está vinculado a um único local. Eles também podem estar interagindo com pessoas próximas, objetos e elementos ambientais que podem distrair sua atenção;

2. Conectividade: A conectividade é muitas vezes lento e não confiável em dispositivos móveis. Isso terá impacto sobre o desempenho de aplicações móveis que utilizam estes características;

3. Tamanho da tela: A fim de proporcionar portabilidade dos dispositivos móveis, o tamanho da tela torna-se um limitador e assim restringe a quantidade informação exibida;

4. Diferentes resoluções de tela: A resolução dos dispositivos móveis é reduzida a partir da área de trabalho de computadores, resultando em imagens de menor qualidade;

\section{Capacidade de processamento limitada: A} fim de proporcionar a portabilidade, dispositivos móveis, muitas vezes contêm menos capacidade de processamento. Isto limitará o tipo de aplicações adequadas para estes dispositivos;

6. Métodos de entrada de dados: Os métodos de entrada disponíveis para dispositivos móveis são diferentes dos utilizados em computadores desktop e exigem um certo nível de proficiência. Este problema aumenta a probabilidade de entrada errada e diminui a taxa de entrada de dados.

\section{Método de pesquisa}

Como método para esta pesquisa estabeleceu-se a utilização de uma revisão sistemática de literatura a objetivar encontrar os estudos que correlacionam os referidos e supracitados campos de estudo. Segundo Sampaio \& Mancini (2007, p. 84) uma revisão sistemática é uma forma de pesquisa que utiliza como fonte de dados a literatura sobre determinado tema. Desta forma entende-se que esse tipo de investigação resulta em um resumo das evidências relacionadas a uma estratégia de intervenção específica.

\subsection{Estratégias adotadas na pesquisa}

A pesquisa teve como estratégia buscar por estudos nas bases Science Direct e que contivessem no título, resumo e palavras-chave os termos typography e usability (tipografia e usabilidade). A fim de se estabelecer um critério ainda maior para o refinamento da pesquisa, buscou-se entre os resultados primários, estudos que fossem focados em dispositivos de interação móvel. Nas bases, a busca foi realizada apenas sobre os campos título, resumo e palavras-chave (title, abstract $\mathrm{e}$

keywords) e o período foi determinado de 2007 até o presente (30 de janeiro de 2017), pois, em se tratando de estudos sobre usabilidade móvel, tem- 


\section{$16^{\circ}$ \\ ERGODESIGN USIHC CINAHPA}

se o ano de 2007 como um marco pois segundo Budiu (2015, não paginado) o lançamento do iPhone estabeleceu uma nova era para este campo de estudo, assim como em 1998 aconteceu com o advento da internet para os computadores (desktop web).

A busca inicial foi realizada na base utilizando os termos definidos na estratégia adotada para a pesquisa, conforme citado anteriormente. Ao todos foram listados 22 estudos relacionados na pesquisa.

Já a busca realizada na base Science Direct resultou que apenas 4 estudos apresentam nos campos título, resumo e palavras-chave os termos que são o foco desta pesquisa, utilizando como parâmetro temporal do ano de 2007 até os dias de hoje (30 de janeiro de 2017).

\subsection{Critérios de avaliação}

Posteriormente foram estipulados parâmetros para refinamento da busca e assim melhorar a inspeção por documentos que contenham, em seu escopo de estudo, os campos em questão (tipografia e usabilidade). Como critérios de exclusão da busca, ficou determinado que:

a) apenas documentos do tipo (document type) artigos (article) seriam listados ao final;

b) documentos repetidos;

c) documentos incompletos;

d) documentos pertinentes às áreas buscadas.

Como resultado obteve-se 13 documentos na e se mantiveram os $\mathbf{4}$ documentos da base Science Direct.

Ao se verificar os títulos dos trabalhos listados na base Scopus, observou-se que o sistema listou o artigo The effect of surface treatment and topography on corrosion behavior of en 1.4404 stainless steel da revista Materials and Corrosion (Materiais e corrosão). Este prontamente foi excluído da listagem.

Em se tratando de estudos repetidos, foram encontrados 2 artigos na base Science Direct que se repetiam na base Scopus, estes foram retirados $16^{\circ}$ Ergodesign - Congresso Internacional de Ergonomia e Usabilidade de Interfaces Humano Tecnológica: Produto, Informações Ambientes Construídos e Transporte

$16^{\circ}$ USIHC - Congresso Internacional de Ergonomia e Usabilidade de Interfaces Humano Computador

CINAHPA | 2017 - Congresso Internacional de Ambientes Hipermídia para Aprendizagem.

da listagem, seguindo os critérios de exclusão estipulados.

Os resultados finais, distribuídos por base, se apresentam nos quadros a seguir:

\begin{tabular}{|c|c|c|c|}
\hline \multicolumn{4}{|c|}{ Estudos da base Scopus } \\
\hline Item & Título & Revista/periódico & Ano \\
\hline 1 & $\begin{array}{l}\text { Effect of typeface, } \\
\text { letter case and } \\
\text { position on } \\
\text { recognition of short } \\
\text { words presented } \\
\text { on-screen }\end{array}$ & $\begin{array}{l}\text { Behaviour and } \\
\text { Information } \\
\text { Technology } \\
\text { 35(6), pp. 442-451 }\end{array}$ & 2016 \\
\hline 2 & $\begin{array}{l}\text { User studies on } \\
\text { Chinese typefaces } \\
\text { and font size for } \\
\text { senior citizens in } \\
\text { Hong Kong } \\
\end{array}$ & $\begin{array}{l}\text { Information Design } \\
\text { Journal } \\
22(3), \text { pp. } 202-220\end{array}$ & 2016 \\
\hline 3 & $\begin{array}{l}\text { AdobeTypeUI: } \\
\text { Projecting a new } \\
\text { OpenType interface }\end{array}$ & $\begin{array}{l}\text { Proceedings of the } \\
\text { 7th Latin American } \\
\text { Conference on } \\
\text { Human Computer } \\
\text { Interaction, CLIHC } \\
2015 \\
2824902 \\
\end{array}$ & 2015 \\
\hline 4 & $\begin{array}{l}\text { Assessing the } \\
\text { impact of typeface } \\
\text { design in a text-rich } \\
\text { automotive user } \\
\text { interface }\end{array}$ & $\begin{array}{l}\text { Ergonomics 57(11), } \\
\text { pp. } 1643-1658\end{array}$ & 2014 \\
\hline 5 & $\begin{array}{l}\text { Toward building } \\
\text { aesthetic, useful } \\
\text { and readable tag } \\
\text { clouds for websites }\end{array}$ & $\begin{array}{l}\text { ICE-B } 2014 \text { - } \\
\text { Proceedings of the } \\
\text { 11th International } \\
\text { Conference on e- } \\
\text { Business, Part of } \\
\text { ICETE } 2014 \text { - 11th } \\
\text { International Joint } \\
\text { Conference on e- } \\
\text { Business and } \\
\text { Telecommunications } \\
\text { pp. 230-235 }\end{array}$ & 2014 \\
\hline 6 & $\begin{array}{l}\text { Visual design } \\
\text { guidelines for } \\
\text { improving learning } \\
\text { from dynamic and } \\
\text { interactive digital } \\
\text { text }\end{array}$ & $\begin{array}{l}\text { Computers and } \\
\text { Education } \\
63 \text {, pp. 248-258 }\end{array}$ & 2013 \\
\hline 7 & $\begin{array}{l}\text { Legible, are you } \\
\text { sure? An } \\
\text { experimentation- } \\
\text { based typographical } \\
\text { design in safety- } \\
\text { critical context }\end{array}$ & $\begin{array}{l}\text { Conference on } \\
\text { Human Factors in } \\
\text { Computing Systems } \\
\text { - Proceedings } \\
\text { pp. } 2287-2296\end{array}$ & 2012 \\
\hline 8 & $\begin{array}{l}\text { Structure extraction } \\
\text { from PDF-based }\end{array}$ & $\begin{array}{l}\text { Proceedings of the } \\
\text { ACM/IEEE Joint }\end{array}$ & 2011 \\
\hline
\end{tabular}



Interfaces Humano Tecnológica: Produto, Informações Ambientes USIHC Construídos e Transporte

$16^{\circ}$ USIHC - Congresso Internacional de Ergonomia e Usabilidade de Interfaces Humano Computador

CINAHPA | 2017 - Congresso Internacional de Ambientes Hipermídia CINAHPA para Aprendizagem.

\begin{tabular}{|c|c|c|c|}
\hline & book documents & $\begin{array}{l}\text { Conference on } \\
\text { Digital Libraries } \\
\text { pp. } 11-20\end{array}$ & \\
\hline 9 & $\begin{array}{l}\text { Visual design of e- } \\
\text { learning } \\
\text { environments }\end{array}$ & $\begin{array}{l}2011 \text { IEEE Global } \\
\text { Engineering } \\
\text { Education } \\
\text { Conference, } \\
\text { EDUCON } 2011 \\
5773192 \text {, pp. } 552- \\
560\end{array}$ & 2011 \\
\hline 10 & $\begin{array}{l}\text { ManiWordle: } \\
\text { Providing flexible } \\
\text { control over wordle }\end{array}$ & $\begin{array}{l}\text { IEEE Transactions } \\
\text { on Visualization and } \\
\text { Computer Graphics } \\
\text { 16(6),5613458, pp. } \\
1190-1197\end{array}$ & 2010 \\
\hline 11 & $\begin{array}{l}\text { The effects of } \\
\text { usability and web } \\
\text { design attributes on } \\
\text { user preference for } \\
\text { e-commerce web } \\
\text { sites }\end{array}$ & $\begin{array}{l}\text { Computers in } \\
\text { Industry } \\
61(4), \text { pp. 329-341 }\end{array}$ & 2010 \\
\hline 12 & $\begin{array}{l}\text { The effect of } \\
\text { aesthetic on the } \\
\text { usability of data } \\
\text { visualization }\end{array}$ & $\begin{array}{l}\text { Proceedings of the } \\
\text { International } \\
\text { Conference on } \\
\text { Information } \\
\text { Visualisation } \\
4272047 \text {, pp. } 637- \\
645\end{array}$ & 2007 \\
\hline
\end{tabular}

Quadro 2 - Resultado da busca inicial da base

\begin{tabular}{|c|l|l|l|}
\hline \multicolumn{4}{|c|}{ Estudos da base Science Direct } \\
\hline Item & Título & Revista/periódico & Ano \\
\hline 1 & $\begin{array}{l}\text { A System Analysis } \\
\text { and Design for } \\
\text { Packaging Design } \\
\text { of Powder Shaped } \\
\text { Fresheners Based } \\
\text { on Kansei } \\
\text { Engineering }\end{array}$ & $\begin{array}{l}\text { Procedia } \\
\text { Manufacturing }\end{array}$ & 2015 \\
\hline 2 & $\begin{array}{l}\text { Effects of screen } \\
\text { resolution and } \\
\text { column ratio on } \\
\text { search performance } \\
\text { and subjective } \\
\text { preferences }\end{array}$ & $\begin{array}{l}\text { Displays } \\
\text { Vol. 33, Issue 1 }\end{array}$ & \\
\hline
\end{tabular}

Quadro 3 - Resultado da busca inicial da base Science Direct

Após a realização de o agrupamento dos trabalhos, o resultado foi de $\mathbf{1 4}$ estudos relacionados às áreas em questão nesta pesquisa.

Para a análise mais aprofundada, ficou estipulado que estudos que possuam como objetos de estudo ou suporte, equipamentos eletrônicos similares aos dispositivos de interação móvel seja pelo aspecto computacional, seja por possuir tela pequena para exibição de informações.

\section{Resultados obtidos}

Aqui são apresentados os estudos que, ao final do refinamento da pesquisa possuam efetivamente correlação com os campos de estudo da tipografia e a usabilidade. Para tal foram analisados os devidos resumos dos trabalhos e assim obteve-se um aprofundamento maior acerca dos estudos listados.

No estudo intitulado Effect of typeface, letter case and position on recognition of short words presented on-screen (PUSNIK; MOZINA; PODLESEK, 2016) os autores apresentam um estudo sobre a combinação de typeface (cara da letra) para a apresentação de informações em televisão. Entende-se que este tipo de estudo não faz parte do campo a que esta pesquisa está investigando pois apresenta como suporte televisões.

Já no estudo intitulado User studies on Chinese typefaces and font size for senior citizens in Hong Kong (KWOK, 2016) apresenta um estudo sobre letras chinesas em três diferentes tamanho de corpo tipográfico apresentaram diferentes performance entre idosos, durante testes de leitura. Este estudo não apresenta o escopo proposto por esta pesquisa por não se tratar de estudos feitos sobre dispositivos de interação móvel.

Bonato \& Bitelo (2015) apresentam em AdobeTypeUI: Projecting a new OpenType interface o avanço tecnológico acerca da melhoria da tipografia digital incluindo diferentes características ao mesmo arquivo de fonte. $\mathrm{O}$ objetivo do estudo foi desenvolver um novo propósito para os arquivos com tecnologia OpenType, seu uso em ferramentas de editoração eletrônica como por exemplo o Adobe InDesign e compreender aspectos básicos do design da interface. Neste artigo foram envolvidos na pesquisa usuários em testes de usabilidade para avaliação dos objetivos propostos. Entende-se que este estudo contempla em parte a proposta desta pesquisa por relaciona de maneira efetiva um 


\section{$16^{\circ}$ \\ ERGODESIGN USIHC CINAHPA}

$16^{\circ}$ Ergodesign - Congresso Internacional de Ergonomia e Usabilidade de Interfaces Humano Tecnológica: Produto, Informações Ambientes Construídos e Transporte

$16^{\circ}$ USIHC - Congresso Internacional de Ergonomia e Usabilidade de Interfaces Humano Computador

CINAHPA | 2017 - Congresso Internacional de Ambientes Hipermídia para Aprendizagem. campo ligado a tipografia e a usabilidade de parâmetros tipográficos ligados a sua manipulação e formatação, porém estes não são destinados a DIM's.

Em Assessing the impact of typeface design in a text-rich automotive user interface (CHAHINE et al., 2014) avaliam o uso de letras 'humanistas' e 'grotescas' em interface automotiva rica em texto. $\mathrm{O}$ estudo identificou que o tipo de letra (typeface) favorece a redução do tempo total da observação entre homens e mulheres. Este apresenta, evidentemente características entre os campos relacionados desta pesquisa, porém, também não está relacionado a dispositivos de interação móvel e sim a interface automotiva.

No estudo Toward building aesthetic, useful and readable tag clouds for websites (DROZDOWSKI et al., 2014) apresenta um estudo acerca de nuvens de etiqueta (tag cloud) como método gráfico para sumarizar documentos de texto ou até web pages. Para isso os autores analisaram páginas de internet que oferecem este tipo de método e que possuam a regras para a configuração da tipografia onde são discutidas posteriormente numa tentativa de quantificar os aspectos estéticos. Este estudo não apresenta relevância pois avalia aspectos visuais, incluindo a tipografia, sob a ótica da estética.

$\mathrm{O}$ estudo Visual design guidelines for improving learning from dynamic and interactive digital text (JIN, 2013) apresenta relevância esta pesquisa pois teve como propósito desenvolver guias sobre design visual a fim de melhorar e aprender a partir do text digital dinâmico e interativo, visto que tais guias derivam da aplicação da tipografia de maneira impressa. Após o desenvolvimento, foram realizados testes para examinar a eficiência dessas guias de design visual. Este estudo atende em parte a esta pesquisa pois correlaciona o uso da tipografia em ambiente digital e utiliza como instrumento de validação testes realizados com usuários, porém não evidencia qual suporte digital é este, podendo ser desktops ou dispositivos móveis.

Em Legible, are you sure? An experimentationbased typographical design in safety-critical context (ANTHÈNES; VINOT, 2012) apresenta um projeto de pesquisa que tem como objetivo criar uma nova typeface para a exibição de informações em interfaces de aviões. São exibidos 3 experimentos usados para a validação das escolhs inicias e como resultado os pesquisadores confirmaram a importância de testes rigorosos com componentes tipográficos como parte da avaliação de textos de saída (output) em sistemas interativos. Entende-se que esse tipo de estudo contempla de maneira abrangente o intuito dessa pesquisa.

No estudo Structure extraction from PDF-based book documents (GAO et al., 2011) os pesquisadores propõem uma nova estrutura computacional para analisar a estrutura física subjacente e a estrutura lógica de arquivos em formato PDF. O termo tipografia aparece como um dos elementos constituintes dessa nova proposta. Entende-se que esse estudo esteja fora do campo desta pesquisa pois se relaciona a estudos de computação aplicada.

\section{Visual design of e-learning environments} (TOMKO; ZAITSEVA, 2011) apresenta os problemas do design visual em ambientes de $e$ learning e seus efeitos sobre o usuário final. $\mathrm{O}$ resumo do artigo não apresenta maiores informações sobre tipografia e testes de validação usuários. Este artigo foi descartado da lista final desta pesquisa.

\section{ManiWordle: Providing flexible control over} wordle (KIM et al., 2010) apresenta uma nova ferramenta de visualização baseada em Wordle que reformula as interações com o layout, suportando manipulações personalizadas. Esta nova ferramenta permite que os usuários manipulem tipografia, cor e composição não apenas em torno do leiaute e sim individualmente por palavra. Ao final é apresentado um estudo de usabilidade comparativo entre as duas ferramentas e que aponta que esta nova ferramenta possui um índice de satisfação maior por parte dos usuários. Acredita-se que este estudo tenha relevância para esta pesquisa apesar de não descrever qual suporte utilizado nas avaliações.

$\mathrm{O}$ artigo The effects of usability and web design attributes on user preference for e-commerce web sites (LEE; KOUBEK, 2010) examina as relações 


\section{$16^{\circ}$ \\ ERGODESIGN USIHC CINAHPA}

entre a usabilidade percebida antes do uso real, tempo de conclusão da tarefa e preferência, e os efeitos de atributos de design na preferência do usuário para sites de comércio eletrônico. Essas descobertas podem ser usadas para construir uma estrutura conceitual para a compreensão das preferências dos usuários e para desenvolver diretrizes de design para produzir sites de comércio eletrônico mais altamente preferidos. Acredita-se que este estudo apresenta dados relativos a referida pesquisa pois visava avaliar aspectos tipográficos, dentre os elementos da interface, com usuários finais de sites de comércio eletrônico.

The effect of aesthetic on the usability of data visualization (CAWTHON; MOERE, 2007) investiga os resultados de um inquérito on-line de 285 participantes, medindo tanto a estética percebida, bem como a eficiência e eficácia das tarefas de recuperação através de um conjunto de 11 diferentes técnicas de visualização de dados. Tais visualizações representam um conjunto de dados hierárquico idêntico, que são normalizados em termos de cor, tipografia e equilíbrio de layout. Este estudo mediu parâmetros como velocidade de conclusão, taxa de precisão, abandono de tarefas e latência de resposta errônea. O estudo demonstra parâmetros previstos no escopo dessa pesquisa e assim contemplam os requisitos para ser considerado relevante.

No artigo A System Analysis and Design for Packaging Design of Powder Shaped Fresheners Based on Kansei Engineering (DJATNA; KURNIATI, 2015) são apresentados análise de embalagens de chá com a premissa de que para apoiar as preferências do cliente e para melhorar as vendas do produto, o design da embalagem deve ser atraente. A principal contribuição desta pesquisa foi identificar os elementos do design das embalagem, identificar o design da embalagem em palavras Kansei e formular o novo design para as mesmas.

$\mathrm{O}$ artigo Effects of screen resolution and column ratio on search performance and subjective preferences refere-se a uma investigação sobre os efeitos da distribuição de proporção no design da página web sobre o desempenho das tarefas do $16^{\circ}$ Ergodesign - Congresso Internacional de Ergonomia e Usabilidade de Interfaces Humano Tecnológica: Produto, Informações Ambientes Construídos e Transporte

$16^{\circ}$ USIHC - Congresso Internacional de Ergonomia e Usabilidade de Interfaces Humano Computador

CINAHPA | 2017 - Congresso Internacional de Ambientes Hipermídia para Aprendizagem. usuário e preferência subjetiva. Sessenta e um participantes foram solicitados a procurar textos nas páginas e o tempo de reação e a taxa de erro foram registrados automaticamente por um software. Apesar desse estudo utilizar um texto, inserido no objeto de estudo (website), os usuários não utilizaram como suporte dispositivos de interação móvel e sim desktops.

Como resultado do estudo analítico obteve-se que alguns dos artigos possuem correlação com os temas propostos (tipografia e usabilidade) porém nenhum deles apresenta como suporte digital, dispositivos de interação móvel, o que configura a falta de exploração científica neste campo.

\section{Considerações finais}

Esta pesquisa objetivou, buscar nas bases e Science Direct, artigos que contivessem estudos que correlacionassem tipografia e usabilidade em dispositivos de interação móvel. Para foi apresentado um referencial que serviu como base para o estudo analítico acerca do escopo dos trabalhos.

Foi realizada uma revisão sistemática na identificação de tais estudos e como resultado da busca obteve-se vinte e dois estudo relacionados. Vale ressaltar que este estudo faz um levantamento preliminar acerca de artigos científicos e não foram levantadas teses e dissertações com abordem os termos pesquisados.

Como sugestão de trabalhos futuros sugere-se que esta pesquisa seja ampliada a outras bases científicas, incluindo teses e dissertações, além da correlação dos termos utilizados nesta pesquisa.

Em seguida foi realizada uma análise em torno dos resumos dos referidos artigos e, ao se aplicar os critérios de exclusão, chegou-se ao resultado que nenhum dos artigos contemplam o objetivo da pesquisa aqui proposta, o que reforça que existe um campo de estudo muito promissor para pesquisas futuras.

\section{Referências bibliográficas}

Realização:

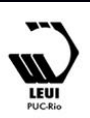




\section{$16^{\circ}$ \\ ERGODESIGN USIHC CINAHPA}

BASTIEN, J. M. C.; SCAPIN, D. L. Ergonomic criteria for the evaluation of human computer interfaces. [s.l.: s.n.], 1993.

BIX, L. The Elements of Text and Message Design and Their Impact on Message Legibility: A Literature Review. Disponível em: $<$ https://scholar.lib.vt.edu/ejournals/JDC/Spring2002/bix.html>. Acesso em: 7 jun. 2016.

BRINGHURST, R. Elementos do estilo tipográfico. 3. ed. São Paulo: Cosac Naify, 2011.

BITELO, C.T.; BONATTO, M.D. AdobeTypeUI: Projecting a new OpenType interface. Proceedings of the 7th Latin American Conference on Human Computer Interaction, CLIHC 2015, [s.v., s.n], Cordoba, 2015.

BUDIU, R. The State of Mobile User Experience. Disponível em: $<$ https://www.nngroup.com/articles/mobileusability-update/>. Acesso em 15 fev. 2017.

BUGGY (LEONARDO ARAÚJO DA COSTA). O MECOtipo: método de ensino de desenho coletivo de caracteres tipográficos. Edição do ed. Recife: [s.n.], 2007.

CAWTHON, N.; MOERE, A. V.. The effect of aesthetic on the usability of data visualization. Proceedings of the International Conference on Information Visualisation, [s.v., s.n.], p. 637-645, Zurich, 2007.

CHAHINE, N. et al.. Assessing the impact of typeface design in a text-rich automotive user interface. Ergonomics, v. 57, n. 11, [s.1.], p. 1643 1658, 2014.

CHIEN-HSIUNG C.; SHU-YING C. Effects of screen resolution and column ratio on search performance and subjective preferences. Displays, v. 33, n. 1, p. 28-35, [s.1.], 2012.

CYBIS, W.; BETIOL, A. H.; FAUST, R.

Ergonomia e usabilidade: conhecimentos, métodos e aplicações. 2. ed. São Paulo: Novatec Editora, 2010.

DROZDOWSKI, M. et al.. Toward building aesthetic, useful and readable tag clouds for websites. Proceedings of the 11th International $16^{\circ}$ Ergodesign - Congresso Internacional de Ergonomia e Usabilidade de Interfaces Humano Tecnológica: Produto, Informações Ambientes Construídos e Transporte

$16^{\circ}$ USIHC - Congresso Internacional de Ergonomia e Usabilidade de Interfaces Humano Computador

CINAHPA | 2017 - Congresso Internacional de Ambientes Hipermídia para Aprendizagem.

Conference on e-Business, Part of ICETE 2014 -

11th International Joint Conference on e-Business and Telecommunications, [s.v., s.n.], p. 230235,Vienna, 2014.

FARIAS, P. L. Notas para uma normatização da nomenclatura tipográfica. In: 6. CONGRESSO BRASILEIRO DE PESQUISA E DESENVOLVIMENTO EM DESIGN, 2004, [S.1.]. Anais... [s.1.:s.n], 2004. v. 1683. Não paginado. CD.

FARIAS, P. L. Tipografia digital: o impacto das novas tecnologias. 4. ed. Teresópolis: 2AB, 2013.

GAO, J. et al. Structure extraction from PDF-based book documents. Proceedings of the ACM/IEEE Joint Conference on Digital Libraries,

GOMES. O design brasileiro de tipos digitais: elementos que se articulam na formação de uma prática profissional. [s.l.] Escola Superior de Desenho Industrial, Universidade do Estado do Rio de Janeiro, 2010.

HAMMERSCHMIDT, C. Tipografia em bulas de medicamento digitais para acesso ao Bulário Eletrônico em dispositivos de interação móvel. [s.l.] Universidade Federal do Paraná, 2014.

HAMMERSCHMIDT, C.; SPINILLO, C Tipografia para leitura em telas de dispositivos de interação móvel: a resolução dos suportes ainda constitui um problema para os designers ?

Congresso Brasileiro de Pesquisa e Desenvolvimento em Design, 2014.

HASLAM, A. O livro e o designer II: como criar e produzir livros. 2. ed. São Paulo: Rosari, 2010. v. 1

HOOBER, S.; BERKMAN, E. Designing Mobile Interfaces. 1. ed. Sebastopol: O'Reilly Media, 2011.

JIN, S. Visual design guidelines for improving learning from dynamic and interactive digital text. Computers and Education, v. 63, [s.n., s.l.], p. 248258, 2013.

KIM, B. et al.. ManiWordle: Providing flexible control over wordle. IEEE Transactions on Visualization and Computer Graphics, v. 6, n. 6, p. 1190-1997, [s.1], 2010.
Realização:
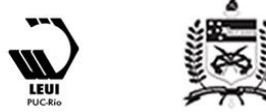


\section{$16^{\circ}$ \\ ERGODESIGN USIHC CINAHPA}

KOUBEK, R. J.; LEE, S.. The effects of usability and web design attributes on user preference for ecommerce web sites. Computers in Industry, v. 61, n. 4, p. 329-341, [s.1.], 2010.

KWOK, B. User studies on Chinese typefaces and font size for senior citizens in Hong Kong.

Information Design Journal, v. 22, n. 2, p. 202-220, 2016.

LUPTON, E. Pensar com tipos. 2. ed. São Paulo: Cosac Naify, 2013.

NIELSEN, J. Usability 101: Introduction to Usability. Disponível em:

$<$ https://www.nngroup.com/articles/usability-101introduction-to-usability/>. Acesso em: 1 jun. 2016.

MOŽINA, K.; PODLESEK, A; PUŠNIK, N. Effect of typeface, letter case and position on recognition of short words presented on-screen. Behaviour and Information Technology, v. 35, n. 6, p. 442-451, [s.1.], 2016.

NIEMEYER, L. Tipografia: uma apresentação. 1. ed. Teresópolis: 2AB, 2010.

REICHENSTEIN, O. Responsive Typography. Disponível em:

$<$ http://informationarchitects.net/blog/responsivetypography/>. Acesso em: 1 jun. 2016.

TOMKO, V.; ZAITSEVA, L. Visual design of elearning environments. 2011 IEEE Global Engineering Education Conference, EDUCON 2011, [s.v., s.n.], p. 552-560, Amman, 2011.

TAUFIK D.; WENNY D. K.. A System Analysis and Design for Packaging Design of Powder Shaped Fresheners Based on Kansei Engineering. Procedia Manufacturing, v. 4, [s.n.], p. 115-123, [s.l.], 2016.

VINOT, J.; ATHÈNES, S.. Legible, are you sure? An experimentation-based typographical design in safety-critical context. Conference on Human Factors in Computing Systems - Proceedings, [s.v., s.n], Austin, 2012.

WAARDE, K. Typographic dimensions and conventional wisdom: a discrepancy? Technical Communication, v. 46, n. November 1997, p. 67- $16^{\circ}$ Ergodesign - Congresso Internacional de Ergonomia e Usabilidade de Interfaces Humano Tecnológica: Produto, Informações Ambientes Construídos e Transporte

$16^{\circ}$ USIHC - Congresso Internacional de Ergonomia e Usabilidade de Interfaces Humano Computador

CINAHPA | 2017 - Congresso Internacional de Ambientes Hipermídia para Aprendizagem.

74, 1999.

ZHANG, D.; ADIPAT, B. Challenges,

Methodologies, and Issues in the Usability Testing of Mobile Applications. International Journal of Human-Computer Interaction, v. 18, n. 3, p. 293308, 2005. 